\title{
Effect of spin-orbit interaction on the optical spectra of single-layer, double-layer, and bulk $\mathrm{MoS}_{2}$
}

\author{
Alejandro Molina-Sánchez, ${ }^{1,2}$ Davide Sangalli, ${ }^{3}$ Kerstin Hummer, ${ }^{4}$ Andrea Marini, ${ }^{3}$ and Ludger Wirtz ${ }^{1,2}$ \\ ${ }^{1}$ Physics and Materials Science Research Unit, University of Luxembourg, L-1511, Luxembourg, Luxembourg \\ ${ }^{2}$ Institute for Electronics, Microelectronics, and Nanotechnology (IEMN), CNRS UMR 8520, Department ISEN, \\ 59652 Villeneuve d'Ascq Cedex, France \\ ${ }^{3}$ Istituto di Struttura della Materia of the National Research Council, Via Salaria Km 29.3, I-00016, Monterotondo Stazione, Italy \\ ${ }^{4}$ Faculty of Physics, Center for Computational Materials Science, University of Vienna, Sensengasse 8, A-1090, Wien, Austria
}

(Received 26 March 2013; published 8 July 2013)

\begin{abstract}
We present converged $a b$ initio calculations of the optical absorption spectra of single-layer, double-layer, and bulk $\mathrm{MoS}_{2}$. Both the quasiparticle-energy calculations (on the level of the GW approximation ) and the calculation of the absorption spectra (on the level of the Bethe-Salpeter equation) explicitly include spin-orbit coupling, using the full spinorial Kohn-Sham wave functions as input. Without excitonic effects, the absorption spectra would have the form of a step function, corresponding to the joint density of states of a parabolic band dispersion in two dimensions. This profile is deformed by a pronounced bound excitonic peak below the continuum onset. The peak is split by spin-orbit interaction in the case of single-layer and (mostly) by interlayer interaction in the case of double-layer and bulk $\mathrm{MoS}_{2}$. The resulting absorption spectra are thus very similar in the three cases, but the interpretation of the spectra is different. Differences in the spectra can be seen in the shape of the absorption spectra at $3 \mathrm{eV}$ where the spectra of the single and double layers are dominated by a strongly bound exciton.
\end{abstract}

DOI: 10.1103/PhysRevB.88.045412

PACS number(s): 73.22.-f, 78.20.Bh, 78.67.Wj

\section{INTRODUCTION}

The promising and interesting physical properties of graphene ${ }^{1}$ have recently stimulated active research in other atomically thin materials, alternative and/or complementary to graphene. ${ }^{2}$ Molybdenum disulfide $\left(\mathrm{MoS}_{2}\right)$, fabricated in its single layer by means of mechanical exfoliation, exhibits a direct band gap of 1.8-1.9 eV, contrary to the indirect band gap of its bulk counterpart. ${ }^{3,4}$ Moreover, single-layer $\mathrm{MoS}_{2}$ has also shown a mobility of $200 \mathrm{~cm}^{2} / \mathrm{Vs}$, which makes it appealing for the design of a new generation of more efficient transistors. ${ }^{5}$ In the field of spintronics, the absence of inversion symmetry in the crystal structure of single-layer $\mathrm{MoS}_{2}$ allows valley polarization by optical pumping with circularly polarized light. ${ }^{6,7}$ This makes possible the design of devices based on spin and valley control. ${ }^{8,9}$ More recently, a remarkable Seebeck coefficient has been measured in single-layer $\mathrm{MoS}_{2}$, opening a new field of application for those materials. ${ }^{10}$ Concerning the optical properties, the photoluminescence in single layers has shown higher efficiency than in multilayers or bulk which is attributed to the direct/indirect band gaps, respectively. The absorption spectra, however, are very similar in all the cases, $3,4,11$ an issue not yet explained. The observed double-peak structure in the absorption spectra can be connected to the splitting of the valence band maximum around the high-symmetry point $K$. For single-layer $\mathrm{MoS}_{2}$, this splitting was explained as a consequence of spin-orbit coupling, which is a result of the missing inversion symmetry. ${ }^{12}$ For bilayer and bulk, this splitting is mainly due to interlayer interaction. ${ }^{13}$ Another open issue is the origin of an additional low-energy peak, presumably caused by excitons bound to surface impurities. ${ }^{14}$

In this context, reliable $a b$ initio calculations of the absorption spectra are necessary for providing the correct interpretation of the reported experimental results. However, the inherent complexity of the Bethe-Salpeter equation ${ }^{15}$ that is usually used to describe the excitonic effects in the optical spectra seems to be the reason for recent inadequately converged calculations. In Ref. 16 the excitonic binding energy was strongly overestimated due to a low k-point sampling and in Refs. 17 and 18 spin-orbit interaction was entirely neglected and the splitting of the excitonic peak was merely due to an unconverged k-point sampling. The aim of our work is to provide well-converged optical spectra, in the framework of the Bethe-Salpeter equation including the effects of spin-orbit coupling. This gives a reliable basis for the interpretation of previous experimental works on single-layer, double-layer, and bulk $\mathrm{MoS}_{2}$. We show that the optical spectrum corresponds essentially to a step function that is the result of the joint density of states for parabolic dispersion in two-dimensional systems. Excitonic effects shift part of the oscillator strength into a discrete excitonic peak below the continuum onset. The splitting of this excitonic peak can be directly related to the splitting of the valence band maximum around $K$ and is thus entirely due to spin-orbit coupling in the case of the single layer and mostly (but not entirely) due to the interlayer interaction for double-layer and multilayer $\mathrm{MoS}_{2}$.

\section{CALCULATION METHODS}

The starting point of the calculation of optical spectra are the Kohn-Sham wave functions and energies calculated with density-functional theory (DFT) in the local-density approximation (LDA). We use the code ABINIT $^{19}$ where wave functions are expanded in plane waves and core electrons are simulated by norm-conserving relativistic pseudopotentials. ${ }^{20}$ The plane-wave energy cutoff is 30 a.u. For Molybdenum, the $4 s$ and $4 d$ semi-core electrons are explicitly calculated (in addition to the $4 d$ and $5 s$ valence electrons). This turns out to be crucial for the proper calculation of the exchange contribution to the self-energy term in the GW calculations. As noted earlier, ${ }^{12,13}$ spin-orbit interaction is important for $\mathrm{MoS}_{2}$ and we calculate the spinor wave functions as input for the following calculations on the level of many-body perturbation theory. 
The inherent underestimation of the band gap given by DFT is corrected with the GW method. ${ }^{15,21}$ We use the nonself-consistent version (denoted as $G_{0} W_{0}$ ) without updating the dielectric function in the screened Coulomb potential $(W)$ or the wave functions and energies in the Green's function $(G)$. These calculations are done with the YAMBO code. ${ }^{22}$ The dielectric function $\epsilon_{G, G^{\prime}}(\omega, q)$ is calculated using the plasmon-pole approximation. ${ }^{23} 50 \mathrm{G}$-vectors are used (for a vacuum distance of 40 a.u. between the periodic images of the single-layer or double-layer calculations). Two hundred unoccupied bands are used in the integration of the self-energy term, yielding converged band-gap corrections for single layer, double layer, and bulk. The k-point sampling is $18 \times 18 \times 1$ for the single and double layers and $18 \times 18 \times 3$ for bulk. The value of the GW correction to the band gap depends on the interlayer distance in the periodic supercells approach. It increases with increasing distance and converges roughly as $1 / d$ (where $d$ is the supercell dimension perpendicular to the layer). This was shown for single layers of hexagonal boron nitride (hBN) ${ }^{24}$ and for single-layers of $\mathrm{MoS}_{2}$ (Ref. 25). At the same time, the excitonic binding energy was also shown to increase roughly as $1 / d$ such that the two effects cancel and the resulting optical spectra hardly depend on the interlayer distance. In our calculations, we use $d=40$ a.u. for the single-layer calculations and of $d=50$ a.u. for the double-layer calculations.

Starting from the Kohm-Sham wave functions and the quasiparticle energies, the optical-spectra are calculated on the level of the Bethe-Salpeter equation (BSE): ${ }^{26-29}$

$$
\left(E_{c \mathbf{k}}-E_{v \mathbf{k}}\right) A_{v c \mathbf{k}}^{S}+\Sigma_{\mathbf{k}^{\prime} v^{\prime} c^{\prime}}\left\langle v c \mathbf{k}\left|K_{e h}\right| v^{\prime} c^{\prime} \mathbf{k}^{\prime}\right\rangle A_{v^{\prime} c^{\prime} \mathbf{k}^{\prime}}^{S}=\Omega^{S} A_{v \mathbf{k} \mathbf{k}}^{S} .
$$

Here, the electronic excitations are expressed in the basis of electron-hole pairs (i.e., vertical excitations at a given $\mathbf{k}$ point from a state in the valence band with quasiparticle energy $E_{v \mathbf{k}}$ to a conduction-band state with energy $E_{c \mathbf{k}}$. The $A_{v c \mathbf{k}}^{S}$ are the expansion coefficients of the excitons in the electron-hole basis and the $\Omega^{S}$ are the eigenenergies (corresponding to the possible excitation energies of the system). If the interaction kernel $K_{e h}$ is absent, Eq. (1) simply yields $\Omega^{S}=\left(E_{c \mathbf{k}}-E_{v \mathbf{k}}\right)$, i.e., the excitations of the system correspond to independent electronhole pairs. The interaction kernel $K_{e h}$ describes the screened Coulomb interaction between electrons and holes, and the exchange interaction, which includes the so-called local fields effect. $K_{e h}$ "mixes" different single-particle excitations, from valence band states $v, v^{\prime}$ to conduction band states $c, c^{\prime}$, giving rise to modified transition energies $\Omega^{S}$ and (possibly) also to discrete excitonic states below the onset of the continuum.

The optical absorption spectrum is given by the imaginary part of the dielectric function, $\varepsilon(\hbar \omega)$, and can be calculated as

$$
\varepsilon_{2}(\hbar \omega) \propto \sum_{S}\left|\sum_{c v \mathbf{k}} A_{v c \mathbf{k}}^{S} \frac{\left\langle c \mathbf{k}\left|p_{i}\right| v \mathbf{k}\right\rangle}{\epsilon_{c \mathbf{k}}-\epsilon_{v \mathbf{k}}}\right| \delta\left(\Omega^{S}-\hbar \omega-\Gamma\right),
$$

where $\langle c \mathbf{k}|\mathbf{p}| v \mathbf{k}\rangle$ are the dipole matrix elements for electronic transitions from valence to conduction states. We consider only light absorption with polarization, and thus the direction of the dipole operator $\mathbf{p}$, in the plane identified by the $\mathrm{MoS}_{2}$ layer. The out-of-plane polarization gives a negligible contribution to absorption at low energies because the local fields, which are strongly inhomogeneous in that direction, shift the oscillator strength to high energies. Excluding phononassisted transitions, the momentum $\mathbf{k}$ is conserved in the absorption process. We use an energy broadening $\Gamma=0.05 \mathrm{eV}$ in all the calculations to mimic the experimental resolution. The BSE calculations have been performed with the code YAMBO. $^{22}$ Since a much higher k-point sampling than for the GW calculations is needed, we use LDA wave functions and energies, corrected by a "scissor' operator obtained from the GW calculations. While this approach does not take into account changes in the valence and conduction band dispersions, we have checked for the excitonic spectrum at a sampling of $18 \times 18 \times 1$ (single layer) that the difference to a BSE calculation based on GW energies is negligible.

\section{QUASIPARTICLE BAND STRUCTURE}

First we study the electronic structure of single-layer, double-layer, and bulk $\mathrm{MoS}_{2}$. It is worth mentioning that an accurate calculation of the exchange interaction in both the GW approximation and the BSE requires the use of semicore ( $4 s$ and $4 p$ ) orbitals for the Mo atoms. The omission of semicore states can lead to an erroneous wave-vector dependence of the GW correction. Furthermore, the spin-orbit interaction has to be taken into account because it removes the degeneracy of the valence band maximum. Single-layer $\mathrm{MoS}_{2}$ (and, in general, an odd number of layers) belongs to the group of symmetry $D_{3 h}$ (Ref. 30), which lacks inversion symmetry. This absence of symmetry, together with the strong spin-orbit coupling of Mo $d$ orbitals, splits the valence band edge at $K$ (Ref. 7). $\mathrm{MoS}_{2}$ with even number of layers, such as the double layer, and the bulk, instead, belong to the symmetry group $D_{6} h$, which does have inversion symmetry. Nevertheless, also in this case a valence band splitting exists but it is caused (predominantly) by the interlayer interaction.

Figure 1 shows the band structure, calculated with LDA and $\mathrm{GW}$, for single-layer, double-layer, and bulk $\mathrm{MoS}_{2}$. The only case where a direct band gap is observed is the single layer, either in LDA or in GW, while for double layer and bulk the band gap is clearly indirect. The origin of this difference is the interlayer interaction. A second minimum in the conduction band lies on the high-symmetry line between $\Gamma$ and $K$ (see vertical dashed lines). For the single layer, this minimum is higher in energy than the one at $K$, but in the double layer and bulk, the interlayer interaction leads to a splitting which shifts the minimum to a lower energy than the minimum at $K$. Moreover, in the double layer and in the bulk, the valence band edge at $\Gamma$ rises higher than the valence band maximum at $\mathbf{K}$. As a consequence the system moves from a direct band gap to an indirect gap semiconductor. The GW corrections are generally larger for single-layer $\mathrm{MoS}_{2}$ then for the double layer and the bulk due to the smaller dielectric screening in the single-layer case.

Our band structures and their interpretation agree very well with the ones of the recent self-consistent GW calculations, ${ }^{13}$ even though the details in the approximations are different: in our study, we do not use self-consistency in the GW calculations which seems to add only a minor energy shift to the $G_{0} W_{0}$ calculation. In turn, we perform the GW calculations with the full spinor wave functions while in Ref. 13 


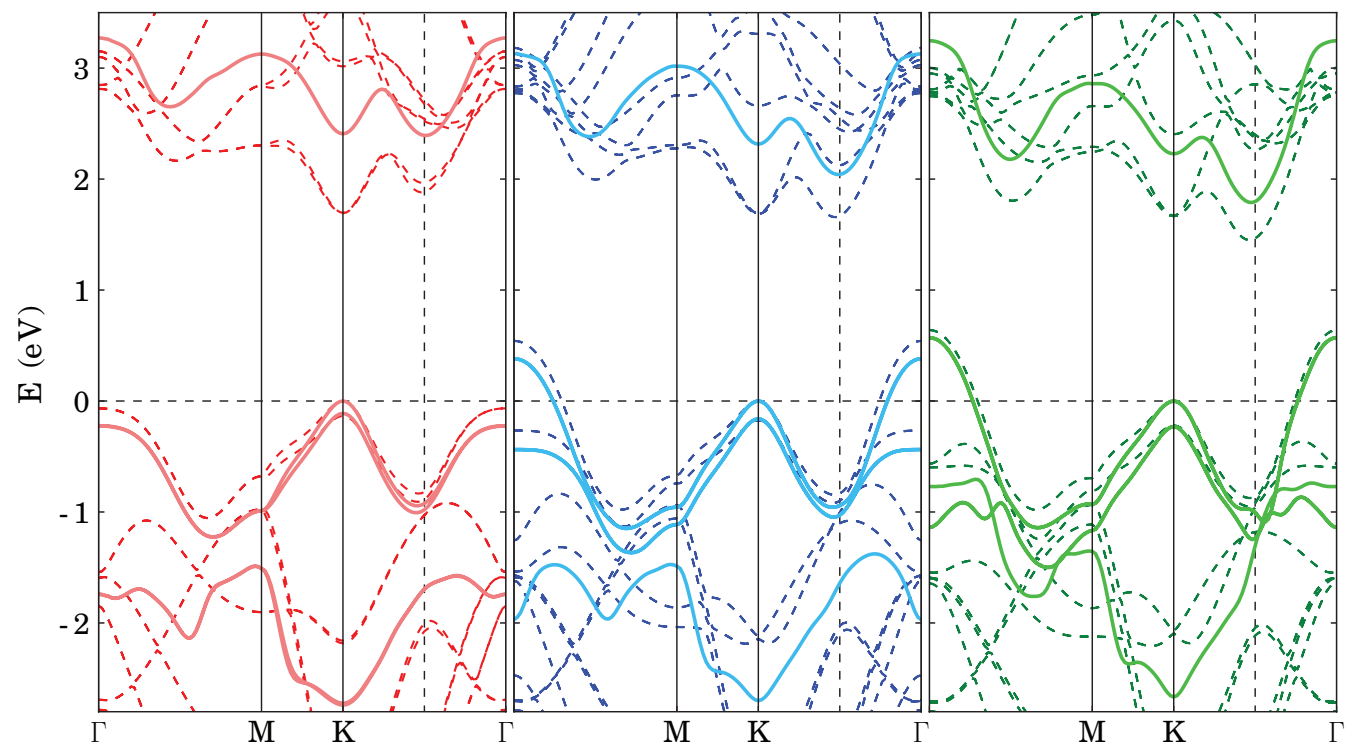

FIG. 1. (Color online) From left to right: Band structure of $\mathrm{MoS}_{2}$ single layer, double layer, and bulk in the LDA (thin dashed lines) and in the $G_{0} W_{0}$ approximation (thick continuous lines).

spin-orbit coupling is introduced after the GW calculations by rediagonalizing the Hamiltonian matrices. The values of the direct band gaps at $K$ are summarized in Table I, combined with the valence band splitting. The values are in reasonable agreement with the ones of Ref. 13. Small differences may be due to the differences in the approximations and from the different values of the cell dimensions.

We remark in this context the importance of the lattice constant. Small variations of this can shift the position of the conduction and valence band edges. Commonly to other GW calculations, we thus use experimental lattice parameters (inplane lattice constant $a=3.15 \AA$ and $c=12.3 \AA$ for bulk) ${ }^{31}$ to avoid artificial strain effects. ${ }^{32} \mathrm{~A}$ similar conclusion was drawn in a theoretical study about the influence of strain on the band gap of $\mathrm{MoS}_{2}$, where strain tends to transform the single-layer $\mathrm{MoS}_{2}$ into an indirect band-gap semiconductor. ${ }^{33,34}$

\section{OPTICAL ABSORPTION SPECTRA}

In the calculation of the optical absorption spectra of $\mathrm{MoS}_{2}$, the convergence with respect to $\mathbf{k}$ sampling is of crucial importance to obtain reliable spectra (see the Appendix). We used a $51 \times 51 \times 1 \mathrm{k}$ point in the case of the single layer and double layer and $21 \times 21 \times 3$ in the case of bulk. Local fields are included in all the calculations. ${ }^{35}$ We show in Fig. 2,

TABLE I. Band gap (in EV) at $K$ point and valence band splitting $\Delta_{v}$ (in $\mathrm{meV}$ ), as obtained in LDA and GW.

\begin{tabular}{lccc}
\hline \hline & \multicolumn{3}{c}{ Material parameters } \\
\cline { 2 - 4 } & Single layer & Double layer & Bulk \\
\hline$E_{K}(\mathrm{LDA})$ & 1.69 & 1.68 & 1.67 \\
$E_{K}(\mathrm{GW})$ & 2.41 & 2.32 & 2.23 \\
$\Delta_{v}(\mathrm{LDA})$ & 134.3 & 173.8 & 220.1 \\
$\Delta_{v}(\mathrm{GW})$ & 112.0 & 160.0 & 230.6 \\
\hline \hline
\end{tabular}

the calculated optical spectra for single-layer, double-layer, and bulk $\mathrm{MoS}_{2}$. The results of the BSE are compared to the optical spectra without the excitonic effects, calculated in the random-phase approximation (RPA, independent-particle picture) using the GW energies [Figs. 2(a) to 2(c)]. The LDA and GW band-gap energies are marked with vertical lines. Finally [Figs. 2(d) to 2(f)] the BSE results are compared to the experimental data. ${ }^{3,4}$ In Figs. 2(d) to 2(f) the theoretical

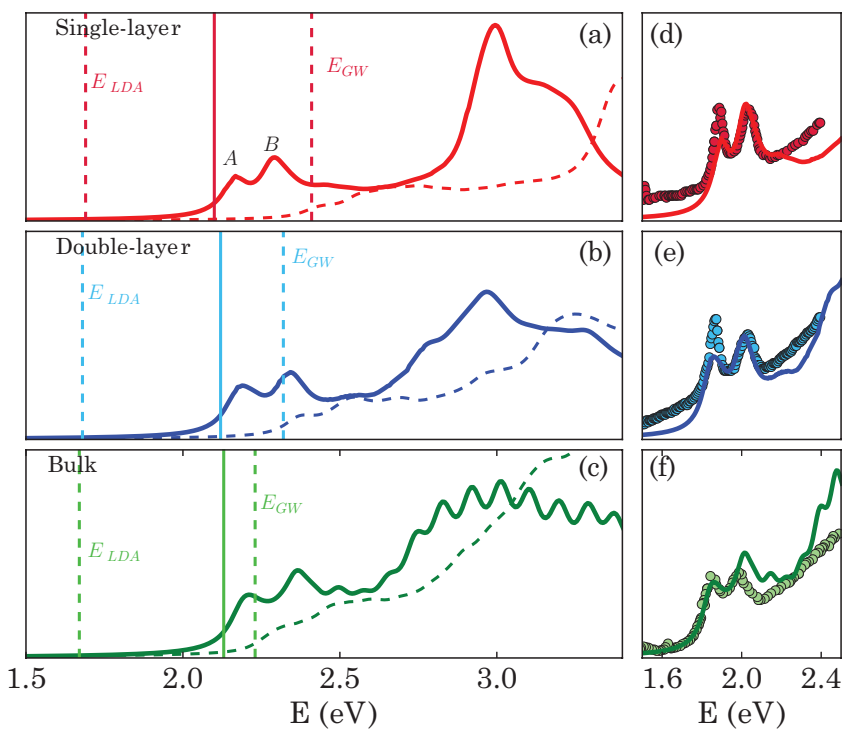

FIG. 2. (Color online) (a)-(c) Optical spectra for single-layer, double-layer, and bulk $\mathrm{MoS}_{2}$, obtained with the BSE (solid lines) and RPA (dashed lines). LDA and GW band gaps at $K$ are marked with vertical dashed lines, the absorption threshold is marked with a vertical solid line. Note that the wiggles above $2.5 \mathrm{eV}$ in panel (c) are due to finite k-point sampling. (d)-(f) Experimental absorption spectra (Refs. 3 and 4 ) in comparison to the calculations (solid lines, shifted by about $-0.2 \mathrm{eV})$. 

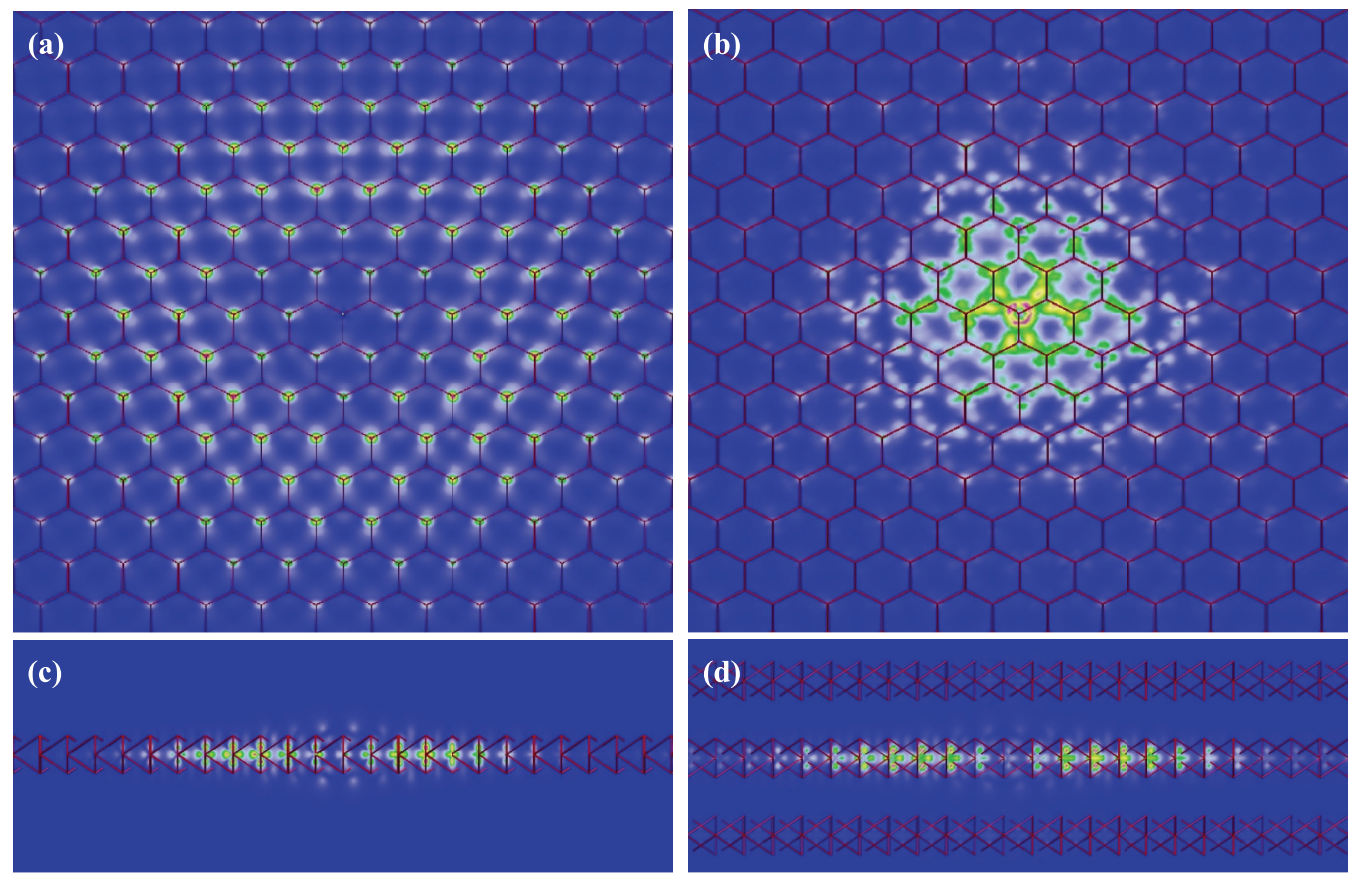

FIG. 3. (Color online) Exciton wave functions: (a) top view of bound exciton $A$ of single layer, (b) top view of bound exciton at $3.0 \mathrm{eV}$ of single layer, (c) lateral view of the exciton $A$ of the single layer, (d) lateral view of the exciton $A$ in bulk. In all cases, the hole has been placed on the Mo atom in the center of the figure. Images were realized with the software XCRYSDEN (Ref. 38).

results are down-shifted by approximately $0.2 \mathrm{eV}$ to compare to the experiments. This discrepancy is in the margin error of common GW and Bethe-Salpeter calculations. Our results give a clear interpretation of the measured absorption spectra, in the low-energy range, and provide reliable predictions within the given accuracy of $\approx 0.2 \mathrm{eV}$, for the higher-energy range, for which no experimental data are available.

The general features of the three optical spectra are very similar, with a double peak structure (denoted as $A$ and $B$ ) at the energy threshold, accompanied by a plateau, and afterwards an abrupt increase of the optical absorption. The RPA spectrum below $2.8 \mathrm{eV}$ resembles in all three cases the sum of two Heaviside step functions. The difference in the two step positions is given by the splitting of the valence band maximum at $K$. This step function profile is the fingerprint of the joint density of states of a two-dimensional (2D) system, with parabolic band structure. "Switching on" excitonic effects preserves the plateau, but, in addition, a split excitonic peak below the onset of the continuum transition occurs. The exciton binding energy is different in the three cases. It is largest for the single layer, where the electron-hole interaction is less screened. The differences in the quasiparticle band gap and excitonic binding energy almost cancel each other ${ }^{24,25}$ and the resulting excitonic peak positions are almost the same in the three cases. The peaks can be directly assigned to the peaks denoted $A$ and $B$ in the experiments. ${ }^{3,4}$ At higher energies, the relevant peak placed at $3.0 \mathrm{eV}$ (close to the blue color in the visible spectrum) in the single layer has larger relative intensity with respect to the peaks $A$ and $B$. Such an intense peak has not yet been reported, as the maximum detection energy in absorption experiments is around $2.4 \mathrm{eV}$ (Ref. 36).

A further inspection of the excitonic eigenvectors allows to assign to each excitonic peak the contributing electron-hole transitions and their location at the Brillouin zone. In all the cases, excitons $A$ and $B$ come from the energy transition at the $K$ point, even when the band gap is indirect. This explains the similarity of the experimental optical absorption. The other relevant peak, at $3.0 \mathrm{eV}$, comes from the part of the Brillouin zone between $K$ and $\Gamma$ (marked with a dashed line in Fig. 1), where we observe a high density of states due to the parallel conduction and valence bands. ${ }^{37}$

To gain further insight of the optical spectra, we represent the excitonic wave functions related to the main peaks of the Bethe-Salpeter spectra. The excitonic wave function can be written as

$$
\left|\Psi^{S}\left(\mathbf{r}_{e}, \mathbf{r}_{h}\right)\right\rangle=\sum_{c v \mathbf{k}} A_{c v \mathbf{k}}^{S} \psi_{c \mathbf{k}}\left(\mathbf{r}_{h}\right) \psi_{v \mathbf{k}}\left(\mathbf{r}_{e}\right),
$$

where $\mathbf{r}_{\mathbf{e}}$ and $\mathbf{r}_{\mathrm{h}}$ are the real-space electron and hole coordinate and $\psi$ the LDA wave functions. The coefficients $A_{c v \mathbf{k}}^{S}$ are obtained by diagonalizing the Hamiltonian of the Bethe-Salpeter equation (with energy $\Omega^{S}$ ). To represent the six-coordinate function, we fix the hole position at the position of a Mo atom and we project onto the function $\left|\Psi^{S}\left[\mathbf{r}_{e}, \mathbf{r}_{h}=(0,0,0)\right]\right|^{2}$ onto the $x-y$ plane. Figure 3(a) shows the exciton wave function of the exciton $A$ of the $\mathrm{MoS}_{2}$ single layer (for double layer and bulk the wave function is essentially identical). This exciton is largely spread, extended over $65 \AA$, in concordance with the small binding energy and with the small effect on the absorption threshold, as reported in the experiments. ${ }^{3,4}$ This also explains the large k-point grid needed to converge the results in the Bethe-Salpeter equation (see the Appendix). The exciton $B$ (not shown here) shows similar trends.

On the contrary, in Fig. 3(b) we observe that the brightest exciton, at $3.0 \mathrm{eV}$ is remarkably localized, being confined to less than $30 \AA$, with a trigonal shape. Among the properties of 
such an exciton we point out the potentially high efficiency of recombination.

Another interesting point (for multilayer and bulk) is to explore if the excitons are confined in one layer or if their wave functions extend over several layers. With this aim we show in Fig. 3(d) the exciton $A$ of the $\mathrm{MoS}_{2}$ bulk. The wave function spreads largely within the plane, but it is undoubtedly constricted to one layer. The density in neighboring layers is negligible and the wave function is very similar to the one of the $A$ exciton in the single layer [Fig. 3(c)]. The large interlayer distance prevents the wave function from spreading to other layers, analogously to what happens in bulk boron nitride..$^{39,40}$

\section{CONCLUSION}

We have performed calculations of the quasiparticle band structure and of the optical absorption spectra of singlelayer, double-layer, and bulk $\mathrm{MoS}_{2}$ including excitonic effects and spin-orbit coupling at the same time. In agreement with previous calculations and with experimental evidence from photoluminescence intensities, ${ }^{3,4,41}$ our $\mathrm{GW}$ calculations demonstrate that only single-layer $\mathrm{MoS}_{2}$ has a direct band gap at $K$. The interlayer interaction makes the band gap indirect for multilayer and bulk $\mathrm{MoS}_{2}$. However, this does not particularly influence the optical absorption spectra in the visible range. These display in all cases a strong excitonic peak (composed of electron-hole pairs around $K$ ) below the onset of continuum transitions which has the step function shape and corresponds to the quasi-2D joint density of states. The layered structure of the material confines the first exciton mostly in a single layer, which causes the similarity of the optical spectra in all the cases. The position of the split excitonic peak is remarkably stable with respect to the number of layers (and with respect to the interlayer spacing in the calculation for single-layer $\mathrm{MoS}_{2}$ ). This has been previously explained as a cancellation effect between the band-gap correction due to the electron-electron interaction and the excitonic binding energy due to the electron-hole interaction. ${ }^{24,25}$ The splitting of the excitonic peak is directly related to the splitting of the valence band maximum around $K$ and is entirely due to spin-orbit coupling for the single layer and (mostly) due to the interlayer interaction for the double layer and bulk. At higher energy (around $3 \mathrm{eV}$ ), the optical spectrum is dominated by electron-hole pairs from transitions around the center of the line $\Gamma \rightarrow K$ where both valence and conduction bands have a minimum and the parallel shape of the bands causes a maximum in the joint density of states. For the single layer a strongly bound exciton causes an additional peak in the spectrum at $3.0 \mathrm{eV}$. For the double layer and bulk, the shape of the spectrum changes and may allow for a spectroscopic distinction between layer numbers.

\section{ACKNOWLEDGMENTS}

The authors acknowledges financial support from the French National Research Agency (ANR) through Project No. ANR-09-BLAN-0421-01. Calculations were done at the IDRIS supercomputing center, Orsay (Project No. 091827),

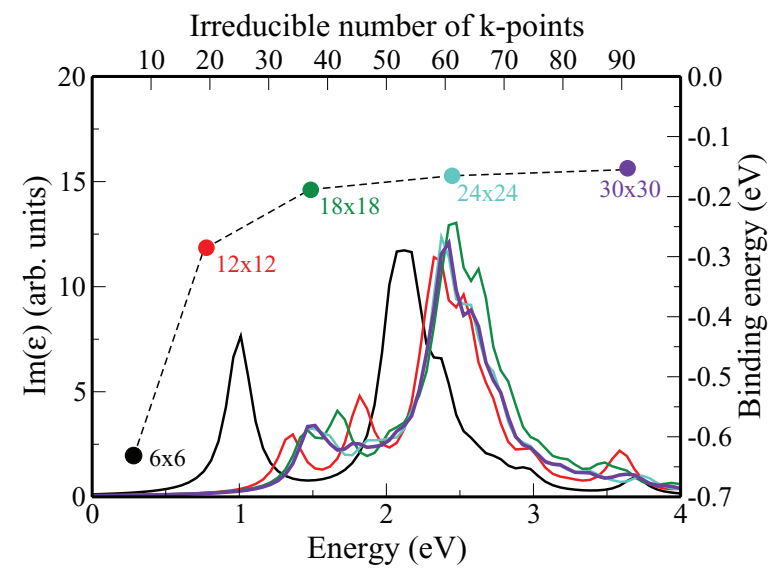

FIG. 4. (Color online) Left axis: Bethe-Salpeter spectra for several k-point grids. Right axis: Exciton binding energy $\left(E_{b}\right)$ as a function of the number of irreducible $\mathbf{k}$ points. Note the correspondence in the colors of the spectra and $E_{b}$.

and at the Tirant Supercomputer of the University of Valencia (group vlc44).

\section{APPENDIX}

The convergence of the optical spectra is a mandatory issue to obtain reliable spectra, comparable with the experimental data. We have performed calculations to check the convergence of the spectra, and in particular of the position of the first excitonic peak with respect to the k-point sampling. A fine sampling can be quite costly in terms of computation time and in some Bethe-Salpeter calculations may reach unexpectedly large values. Figure 4 shows the Bethe-Salpeter spectra of the $\mathrm{MoS}_{2}$ single layer for several $\mathbf{k}$ grids (for simplicity we have omitted the spin-orbit coupling). Additionally, we have represented the exciton binding energy, $E_{b}$, as a function of the number of irreducible $\mathbf{k}$ points, marking in each case the corresponding grid. We observe the slow convergence of the first excitonic peak, not reached before a $18 \times 18$ sampling. Moreover, for small grids some secondary and artificial peaks appear in the spectrum and the underlying Heaviside function of the optical absorption is not reached unless dense grids are used $(30 \times 30)$. This value is considerably larger than the one used in Ref. 16 where only a $6 \times 6$ grid was used, leading to isolated peaks in the absorption spectra where the density of states predicts a plateau following the excitonic peak. Our test calculations also explain why in Refs. 17 and 18 split excitonic peaks were observed, even though spin-orbit coupling was not included.

The exciton binding energy $E_{b}$ also needs at least an $18 \times 18$ grid to be converged within $0.1 \mathrm{eV}$. It is worth noting that the second excitonic peak (the most intense), located at $2.5 \mathrm{eV}$ converges much faster, approximately for a $12 \times 12$ grid. The difference in behavior of those excitons can be better understood by an inspection of their wave functions, shown in Figs. 3(a) and 3(b). In the case of the first peak, the wave function is very spread, more than 18 unit cells in each direction on the plane. We remind that an $n \times n$ grid in the reciprocal space allows only to map the exciton wave function in the real-space $n \times n$ unit cell, therefore, inadequately small 
grids lead to an artificial confinement of the exciton which increases its binding energy. This argument also clarifies why the second exciton converges much faster, its wave function being confined within a few unit cells.
${ }^{1}$ K. Novoselov, D. Jiang, F. Schedin, T. Booth, V. Khotkevich, S. Morozov, and A. Geim, Proc. Natl. Acad. Sci. USA 30, 10451 (2005).

${ }^{2}$ J. N. Coleman, M. Lotya, A. O’Neill, S. D. Bergin, P. J. King, U. Khan, K. Young, A. Gaucher, S. De, R. J. Smith, I. V. Shvets, S. K. Arora, G. Stanton, H. Y. Kim, K. Lee, G. T. Kim, G. S. Duesberg, T. Hallam, J. J. Boland, J. J. Wang, J. F. Donegan, J. C. Grunlan, G. Moriarty, A. Shmeliov, R. J. Nicholls, J. M. Perkins, E. M. Grieveson, K. Theuwissen, D. W. McComb, P. D. Nellist, and V. Nicolosi, Science 331, 568 (2011).

${ }^{3}$ A. Splendiani, L. Sun, Y. Zhang, T. Li, J. Kim, C. Y. Chim, G. Galli, and F. Wang, Nano Lett. 10, 1271 (2010).

${ }^{4}$ K. F. Mak, C. Lee, J. Hone, J. Shan, and T. F. Heinz, Phys. Rev. Lett. 105, 136805 (2010).

${ }^{5}$ B. Radisavljevic, A. Radenovic, J. Brivio, V. Giacometti, and A. Kis, Nat. Nanotech. 6, 147 (2011).

${ }^{6}$ W. Yao, D. Xiao, and Q. Niu, Phys. Rev. B 77, 235406 (2008).

${ }^{7}$ D. Xiao, G. B. Liu, W. Feng, X. Xu, and W. Yao, Phys. Rev. Lett. 108, 196802 (2012).

${ }^{8}$ H. Zeng, J. Dai, W. Yao, D. Xiao, and X. Cui, Nat. Nanotech. 7, 490 (2012).

${ }^{9}$ K. F. Mak, K. He, J. Shan, and T. F. Heinz, Nat. Nanotech. 7, 494 (2012).

${ }^{10}$ M. Buscema, M. Barkelid, V. Zwiller, H. S. J. van der Zant, G. A. Steele, and A. Castellanos-Gomez, Nano Lett. 13, 358 (2013).

${ }^{11}$ A. Castellanos-Gomez, M. Barkelid, A. M. Goossens, V. E. Calado, H. S. J. van der Zant, and G. A. Steele, Nano Lett. 12, 3187 (2012).

${ }^{12}$ Z. Y. Zhu, Y. C. Cheng, and U. Schwingenschlögl, Phys. Rev. B 84, 153402 (2011).

${ }^{13}$ T. Cheiwchanchamnangij and W. R. L. Lambrecht, Phys. Rev. B 85, 205302 (2012).

${ }^{14}$ T. Korn, S. Heydrich, M. Hirmer, J. Schmurtzler, and C. Schüller, Appl. Phys. Lett. 99, 102109 (2011).

${ }^{15}$ G. Onida, L. Reining, and A. Rubio, Rev. Mod. Phys. 74, 601 (2002).

${ }^{16}$ A. Ramasubramaniam, Phys. Rev. B 86, 115409 (2012).

${ }^{17}$ N. Singh, G. Jabbour, and U. Schwingenschlögl, Eur. Phys. J. B 85, 392 (2012).

${ }^{18}$ J. Feng, X. Qian, C.-W. Huang, and J. Li, Nat. Photon. 6, 866 (2012).

${ }^{19}$ X. Gonze et al., Comput. Mater. Sci. 25, 478 (2002).

${ }^{20}$ C. Hartwigsen, S. Goedecker, and J. Hutter, Phys. Rev. B 58, 3641 (1998).
${ }^{21}$ L. Hedin and S. Lundqvist, Solid State Phys. 23, 1 (1970).

${ }^{22}$ A. Marini, C. Hogan, M. Grüning, and D. Varsano, Comput. Phys. Commun. 180, 1392 (2009); http://www.yambo-code.org.

${ }^{23}$ The exact position of the pole in this model is not important. Moving the pole leads to differences of less than $5 \mathrm{meV}$.

${ }^{24}$ L. Wirtz, A. Marini, and A. Rubio, Phys. Rev. Lett. 96, 126104 (2006).

${ }^{25}$ H. P. Komsa and A. V. Krasheninnikov, Phys. Rev. B 86, 241201(R) (2012).

${ }^{26}$ G. Strinati, Phys. Rev. Lett. 49, 1519 (1982).

${ }^{27}$ G. Strinati, Phys. Rev. B 29, 5718 (1984).

${ }^{28}$ M. Rohlfing and S. G. Louie, Phys. Rev. B 62, 4927 (2000).

${ }^{29}$ M. Palummo, O. Pulci, R. Del Sole, A. Marini, P. Hahn, W. G. Schmidt, and F. Bechstedt, J. Phys.: Condens. Matter 16, S4313 (2004).

${ }^{30}$ A. Molina-Sánchez and L. Wirtz, Phys. Rev. B 84, 155413 (2011).

${ }^{31}$ N. Wakabayashi, H. G. Smith, and R. M. Nicklow, Phys. Rev. B 12, 659 (1975).

${ }^{32}$ P. Rinke, M. Winkelnkemper, A. Qteish, D. Bimberg, J. Neugebauer, and M. Scheffler, Phys. Rev. B 77, 075202 (2008).

${ }^{33}$ L. F. Mattheiss, Phys. Rev. B 8, 3719 (1973).

${ }^{34}$ W. S. Yun, S. W. Han, S. C. Hong, I. G. Kim, and J. D. Lee, Phys. Rev. B 85, 033305 (2012).

${ }^{35}$ To speed up the calculation to obtain smooth spectra, we have also tested the double-grid method proposed by the authors of Ref. 42. In this case a basic grid of $21 \times 21 \times 1$ for the single layer and double layer yields almost the same results as the $51 \times 51 \times 1$ grid.

${ }^{36}$ G. Plechinger, F.-X. Schrettenbrunner, J. Eroms, D. Weiss, C. Schüller, and T. Korn, Phys. Status Solidi RRL 6, 126 (2012).

${ }^{37}$ M. Cardona and P. Y. Yu, Fundamentals of Semiconductors (Springer-Verlag, Berlin, 1996).

${ }^{38}$ A. Kokalj, Comput. Mater. Sci. 28, 155 (2003); http://www.xcrysden.org/.

${ }^{39}$ B. Arnaud, S. Lebègue, P. Rabiller, and M. Alouani, Phys. Rev. Lett. 96, 026402 (2006).

${ }^{40}$ S. Galambosi, L. Wirtz, J. A. Soininen, J. Serrano, A. Marini, K. Watanabe, T. Taniguchi, S. Huotari, A. Rubio, and K. Hämäläinen, Phys. Rev. B 83, 081413(R) (2011).

${ }^{41}$ G. Sallen, L. Bouet, X. Marie, G. Wang, C. R. Zhu, W. P. Han, Y. Lu, P. H. Tan, T. Amand, B. L. Liu, and B. Urbaszek, Phys. Rev. B 86, 081301(R) (2012).

${ }^{42}$ D. Kammerlander, S. Botti, M. A. L. Marques, A. Marini, and C. Attaccalite, Phys. Rev. B 86, 125203 (2012). 